\title{
«Haciendo de la coordinación una experiencia transversal: el estudio de la Estructura Económica en el siglo XXI desde la perspectiva del largo plazo»
}

\author{
Fernández Sánchez, Pedro ${ }^{a}$ y Hurtado Ocaña, Inmaculada ${ }^{b}$ \\ aProfesor Adjunto, Departamento de Economía, Universidad CEU San Pablo (fersan.fcee@ceu.es), \\ bProfesora Titular, Departamento de Economía, Universidad CEU San Pablo (huroca@ceu.es)
}

\section{Abstract}

CEU San Pablo University, and more specifically its Faculty of Economic and Business Sciences, decided to launch a subject that involves the compendium and coordination of two clearly differentiated subjects: Economic History and Economic Structure. This has meant, not only the definition of a single program that integrates all the knowledge of both subjects, but also that two lecturers teach the same subject in the same group. Thus, in Structure and Economic History there is a complete and total coordination of the subjects under study, which allows students to link the knowledge, acquired in both of them. Furthermore, this allows them to develop critical thinking, and to face the analysis of the main economic problems from different perspectives.

Keywords: horizontal coordination, vertical coordination, transversality, interdisciplinarity

\section{Resumen}

La Universidad CEU San Pablo, y más concretamente su Facultad de Ciencias Económicas y Empresariales, ha apostado por poner en marcha una asignatura que supone el compendio y coordinación de lo que hasta ahora han sido dos materias claramente diferenciadas: Historia Económica y Estructura Económica. Esto ha supuesto no sólo la definición de un único programa que integra todos los conocimientos propios de ambas materias, sino también que dos profesores impartan en el mismo grupo la misma asignatura. De esta forma en Estructura e Historia Económica se da una plena y total coordinación de los temas objeto de estudio, lo que permite a los estudiantes relacionar los conocimientos adquiridos en dos materias diferentes. En última instancia esto les permite desarrollar un pensamiento crítico, y a afrontar el análisis de los principales problemas económicos desde diferentes perspectivas.

Palabras clave: coordinación horizontal, coordinación vertical, transversalidad, interdisciplinariedad.

\section{Introducción}

El presente proyecto pedagógico corresponde a la asignatura «Historia y Estructura Económica» que se imparte en la Facultad de Ciencias Económicas y Empresariales en el 
segundo curso del Grado de Marketing y Gestión Comercial y en el doble grado de Publicidad y Relaciones Públicas y Márketing. La peculiaridad de la asignatura es que se engloba dentro de dos Áreas de Conocimiento, Historia e Instituciones Económicas y Estructura Económica, pertenecientes ambas al Departamento de Economía. Supone un claro ejemplo de innovación docente en tanto en cuanto integra áreas de conocimiento que, si bien son afines, presentan objetivos distintos y además permiten a los estudiantes adquirir competencias complementarias. Se circunscribe dentro de una estrategia de actuación docente que permite unir el aprendizaje académico con el natural, descrito por Fernández Batanero (2004), contribuyendo mediante esta transversalidad curricular a que los estudiantes encuentren sentido al trabajo que realizan.

El objetivo que nos planteamos con la puesta en marcha de esta asignatura es doble: por un lado, coordinar dos materias en una, lo que implica que los profesores que imparten la asignatura son dos, cada uno especialista en su campo; y por otro lado, enseñar a los alumnos a extraer las lecciones básicas de un tema para poder extrapolar sus efectos en las diferentes áreas a las que afecta. Este doble objetivo implica, no sólo que los profesores deben llevar a cabo una coordinación horizontal entre ellos de forma plena y completa; sino que además los alumnos han de saber tanto trabajar de forma autónoma como en grupos de trabajo (especialmente para la realización de las prácticas de la asignatura). Con ello se pretende dar una mejor formación al estudiante, para poder hacer frente a las necesidades presentes y futuras de la sociedad, ya que tal y como se estableció en la Declaración Mundial sobre la Educación Superior en el siglo XXI, son funciones de la misma no sólo la educación, sino también la formación y la investigación, constituyendo uno de sus objetivos formar profesionales cualificados pero además ciudadanos responsables ${ }^{1}$. Desde esta perspectiva nuestra propuesta se alinea plenamente con la Estrategia Universidad puesta en marcha en España, que considera que la educación superior es un servicio público cuyo objetivo es contribuir a la modernización de la sociedad mediante la introducción de nuevas ideas y el fomento del espíritu crítico de los estudiantes. La concepción de la asignatura, por tanto, tiene por objeto esa formación integral del alumno, no sólo en conocimientos sino también en otras competencias $^{2}$. De hecho, la mayor parte de los informes internacionales ponen de relieve que una formación universitaria que obvia las competencias transversales (las que esta experiencia docente precisamente trata de potenciar), resulta incompleta, impidiendo un desarrollo óptimo de los estudiantes tanto en el terreno personal como en el profesional ${ }^{3}$.

Todos los actores implicados en el sistema educativo, por consiguiente, deben llevar a cabo una participación activa en el desarrollo de la clase, ya que la asignatura supone la plena integración de las dos áreas de conocimiento. Se pretende con ello variar el sentido tradicional de las clases: el profesor ya no sólo transmite conocimientos, sino que guía al alumno para que adquiera competencias que le permitan complementar, argumentar e

\footnotetext{
${ }^{1}$ Cátedra UNESCO (2000)

${ }^{2}$ European Commission (2018:22)

${ }^{3}$ Véanse las recomendaciones de la OIT (2017) o CEDEFOP (2014). De hecho, ya la ANECA en su Libro Blanco de 2005, exponía no sólo la necesidad de producir y transmitir conocimientos dentro de las Universidades, sino también de que dichos conocimientos permitan una formación integral de cara a la inserción laboral de los estudiantes.
} 
integrar los conocimientos adquiridos en el largo plazo. Esto permitirá a los estudiantes, en un futuro, un mejor desenvolvimiento en un mercado laboral cada vez más competitivo. No en vano lo que se persigue con la transversalidad universitaria es una formación para la vida, y esta es precisamente la filosofía que ha guiado el diseño y puesta en práctica de la asignatura Estructura e Historia Económica ${ }^{4}$.

Por otra parte, la concepción y estructura metodológica de la materia, impartida por dos profesores pertenecientes a cada una de las áreas, exige no sólo una mayor interacción entre profesor y alumno, sino también entre los propios alumnos y entre ambos profesores. Supone la introducción, por tanto, de una nueva cultura de trabajo en las Áreas de Conocimiento que tiene por objeto motivar al estudiante, fomentando su autonomía y creatividad ${ }^{5}$.

A continuación, se detalla la organización de la asignatura, principal innovación docente derivada de la coordinación horizontal de las tradicionales materias de Economía Española e Historia Económica. Se expone el programa de la asignatura, así como la metodología docente empleada y la evaluación de las competencias. En el segundo apartado se ponen de relieve los principales aspectos que hacen de este proyecto docente algo novedoso, que implica un claro avance en el desarrollo de una materia universitaria. Se incluyen además los principales resultados y conclusiones de esta experiencia docente.

\section{1. "Historia y Estructura Económica" o cómo hacer de la coordinación entre materias una asignatura dinámica}

Tradicionalmente, las asignaturas de Historia Económica y de Estructura Económica pertenecen a dos campos tan amplios de la Ciencia Económica que se han impartido en todas las Facultades de Economía como materias independientes. Con la puesta en marcha de nuevos Grados en la Facultad de Ciencias Económicas y Empresariales de la Universidad CEU San Pablo, se decidió proceder a la integración de ambas materias - Historia y Estructura Económica - dando un paso más en la coordinación y transversalidad ${ }^{6}$. Se imparte en el Grado de Marketing y Gestión Comercial y en el doble grado de éste con Publicidad y Relaciones Públicas, lo que supone un gran reto dado el perfil del alumno que opta por estos grados, con un carácter mucho más empresarial y creativo y más alejado de las asignaturas propias de la Ciencia Económica, como son las que nos ocupan. Se pretendía avanzar en el principio de complementariedad o mutua compleción, propio de la coordinación entre materias del mismo o distinto departamento, impartidas en el mismo curso $^{7}$.

La asignatura centra su objetivo en el análisis del funcionamiento y las características básicas de la economía española en el contexto mundial, así como los principales problemas a los

\footnotetext{
${ }^{4}$ De la Herrán (2005:247)

${ }^{5}$ Fernández Batanero (2004)

${ }^{6}$ La Facultad de Ciencias Económicas y Empresariales de la Universidad CEU San Pablo puso en marcha en su momento varias experiencias piloto que ayudaban a entender con mayor profundidad la transversalidad y la coordinación vertical y horizontal, siguiendo las líneas fijadas por el Espacio Europeo de Enseñanza Superior (Arroyo, Hurtado, Abeleira y Requejo, 2011).

${ }^{7}$ De la Herrán (2005:242)
} 
que ésta se enfrenta en un entorno cada vez más globalizado. La base temporal de la que se parte no es el corto-medio plazo, sino la evolución a lo largo de todo el siglo XX y los años del siglo XXI ya transcurridos. De esta forma, el programa de la asignatura ha quedado definido como se especifica a continuación:

Tema 1. Los condicionantes naturales

Tema 2. Población y Recursos Humanos

Tema 3. Producto y renta por habitante

Tema 4. Estructura Sectorial de la Economía Española

Tema 5. España dentro de Europa: atraso y convergencia de la economía española

Tema 6. La evolución económica de las regiones españolas

Como se desprende del programa, no existe una diferenciación entre Historia y Estructura Económica. Cada tema es abordado por ambos profesores, que deben coordinarse perfectamente para ir dándose paso uno a otro cuando corresponda. De esta forma, los estudiantes no perciben que las áreas de conocimiento son estancas y diferenciadas (como ocurriría si el programa quedara divido en dos partes), sino que, pueden ir viendo como los problemas o rasgos del pasado marcan o condicionan las actuaciones del presente.

La metodología docente empleada para conseguir este objetivo viene determinada por las directrices marcadas por el EEES. Sin embargo, y dada la peculiaridad de la materia, se ha adaptado a sus necesidades específicas. Así, la metodología docente constituye un proyecto pedagógico doblemente creativo, que se aparta de lo que tradicionalmente se ha seguido en este tipo de asignaturas. Dicha creatividad se observa no sólo en la interacción entre los dos profesores encargados de la materia, sino también en la interacción entre ellos y el alumnado con el desarrollo de las clases y de las tutorías de forma compartida. La presencialidad se lleva a cabo a través de clases teóricas, prácticas y tutorías. Dentro de las clases teóricas se puede diferenciar a su vez entre Clases Magistrales y Seminarios Teóricos.

Las Clases Magistrales, un total de tres a lo largo del semestre, son impartidas de forma alterna por cada uno de los profesores. Su diseño poco tiene que ver con las tradicionales Lecciones Magistrales. En este tipo de clases se exponen los aspectos más teóricos de la asignatura, que permiten al alumno obtener una visión de conjunto que se desarrollará posteriormente en los seminarios. La novedad de las Clases Magistrales de Historia y Estructura Económica es que rompen con el hábito de que el alumno es un mero oyente y escriba, y se convierte en parte esencial de la misma. Para ello, los profesores facilitan previamente todo el material necesario para su preparación (materiales didácticos de apoyo como artículos de máxima actualidad que se difundirán a través del portal del alumno o bibliografía documental de suficiente relevancia para su estudio detallado). El profesor en este tipo de clase sólo aborda los aspectos fundamentales de la materia, dando pie a que el alumno pueda participar activamente en desarrollo de los contenidos. En este sentido, este nuevo sistema permite que en la Clase Magistral el profesor no sea el único protagonista y con ello se incentiva que el alumno participe en el proceso de aprendizaje, planteando algunas preguntas clave que obliguen a los estudiantes a leer, interpretar y exponer su particular 
visión de los hechos. Tres son las Clases Magistrales que se imparten a lo largo del Semestre insertas en el programa anteriormente detallado: $1^{\text {a }}$ Clase Magistral, El crecimiento económico en el largo plazo, $2^{\mathrm{a}}$ Clase Magistral, Comercio y globalización económica y $3^{\mathrm{a}}$ Clase Magistral, La Unión Europea como proceso de integración regional

Al mismo tiempo, se ponen en marcha diversos Seminarios para tratar los temas del programa de la asignatura, que los alumnos tendrán que preparar bajo la dirección de los profesores a partir de una bibliografía recomendada y seleccionada para cada uno de ellos. Los alumnos deben exponer oralmente las principales conclusiones extraídas de los documentos analizados para su posterior debate en clase.

Este tipo de clases teóricas tiene su complemento en las clases prácticas. Para ello, los alumnos matriculados se dividen en grupos de trabajo que han de analizar y manejar con fluidez los indicadores cuantitativos fundamentales de la economía, de tal forma que sepa interpretar los datos regionales y pueda hacer una comparativa con las explicaciones que se han hecho en clase a nivel nacional, y que además pueda interactuar entre los distintos grupos formados para que contrasten los resultados obtenidos. Con ello se pretende acabar con la tradicional separación entre teoría y práctica, fomentando la interpretación de la práctica desde la teoría y de iluminar la teoría con la propia práctica ${ }^{8}$.

Para una mejor atención a los alumnos, la clase se divide en dos grupos, que dirigen cada uno de los profesores responsables de la asignatura, así, se obtiene un mejor funcionamiento y una explicación más personalizada. Las clases prácticas son las siguientes:

Clase Práctica Introductoria. Explicación de cómo se hace un trabajo de investigación. Utilización de las principales bases de datos de la economía española en el aula de informática. Esto permitirá al alumno familiarizarse con el uso de las nuevas tecnologías aplicadas a la economía que necesitará en las posteriores clases prácticas.

Práctica 1. Población y Mercado Laboral en España

Práctica 2. Producto y Renta por habitante

Práctica 3. Estructura sectorial de la economía española

Práctica 4. Convergencia y divergencia en el largo plazo

Práctica 5. La evolución económica de las regiones españolas

El objetivo de estas clases prácticas es que los alumnos de la Universidad San Pablo puedan ser capaces, cuando se incorporen al mercado laboral, de buscar la información, interpretarla, trabajar en grupo, respetar las opiniones discrepantes y asumir un calendario estricto de entregas.

Con respecto a las tutorías siempre han constituido una seña de identidad de la Universidad CEU San Pablo. En el caso de esta asignatura constituyen el puente entre la presencial y la no presencial. A través de las nuevas tecnologías el alumno puede estar en continuo contacto con el profesor para resolver cualquier tipo de dudas que le surjan en el estudio de la materia

\footnotetext{
${ }^{8}$ Fernández Batanero (2004)
} 
o en el desarrollo de las prácticas. De hecho, son el canal que está permitiendo el feedback entre alumnos y profesores para que ellos puedan sugerir temas de actualidad relativos a la materia a tratar en las clases. En este sentido la Blackboard, se convierte en una piedra angular de este proyecto docente. No sólo es importante para el desarrollo de las tutorías, sino también para el correcto desenvolvimiento de las clases teóricas y de los seminarios prácticos. Todos los materiales, clases y artículos de actualidad relacionados con la materia, estarán a disposición del alumno a través de la Blackboard. Cualquier aportación de los alumnos, de material didáctico que pueda ser de interés para sus compañeros, también será colgado para que todos tengan acceso al mismo.

Con esta metodología docente se persigue que los alumnos adquieran unas competencias que les diferencien del resto de los universitarios en el mercado laboral. En concreto, se busca que los estudiantes demuestren poseer y comprender los conocimientos en un área de estudio tan dinámica y de actualidad como es la economía española y su transformación en el largo plazo. No sólo eso, sino que, además, tengan la capacidad de reunir e interpretar datos relevantes y extrapolarlos a otras áreas de conocimiento para emitir juicios, que incluyan una reflexión sobre temas relevantes de índole social, científica o ética. Y que esos juicios valorados de forma personal puedan ser transmitidos, tanto a un público especializado como no especializado, respetando opiniones discrepantes y aprendiendo de las valoraciones de los demás. De esta forma, las capacidades de nuestros estudiantes destacarán en la sociedad por el respeto a todas las ideas; por el orgullo ante el saber auto aprendido y transmitido; y por el esfuerzo personal.

¿Cómo se evalúa la adquisición de estos conocimientos y competencias por parte del estudiante? Los objetivos de conocimiento se valoran mediante un ejercicio escrito de preguntas de razonamiento y tipo test en las fechas oficiales de la convocatoria ordinaria. Sólo se tiene en cuenta la evaluación continua a aquellos alumnos que han asistido regularmente a clase, (todos aquellos cuya asistencia es superior al 75\%), pues como ya se ha apuntado se trata de una asignatura presencial. La valoración de todas las pruebas de la evaluación continua supone el $50 \%$ de la calificación final ordinaria y el otro $50 \%$ se corresponde con la nota obtenida en el examen final del semestre. La evaluación continua consiste en la realización de un control (que supone el $20 \%$ de la nota total) y las prácticas conjuntas relativas a las prácticas detalladas anteriormente (con una valoración de un 30\%). Estas prácticas se presentan en varios trabajos escritos que además han de ser defendidos por los estudiantes ante sus compañeros. En la convocatoria ordinaria se exige a los alumnos que han de alcanzar, como mínimo, un 3,5 sobre 10 tanto en el examen final como en la media de prácticas, para garantizar la adquisición de las competencias mínimas.

Gracias a este diseño los estudiantes de la asignatura Estructura e Historia Económica adquieren la mayor parte de las competencias transversales consideradas clave por Martínez Clares y González Morga (2019: 9). Agrupadas por las autoras en función del modelo Tuning, posteriormente empleado en la elaboración de los Libros Blancos de las titulaciones universitarias españolas por la Agencia Nacional de Evaluación de la Calidad y Acreditación 
(ANECA), los alumnos de esta asignatura adquieren 14 de las 17 competencias transversales propuestas ${ }^{9}$.

Tabla 1.- Escala de competencias transversales adquiridas por los estudiantes de la asignatura de Estructura e Historia Económica

\begin{tabular}{|l|}
\hline \multicolumn{1}{|c|}{ Competencias Instrumentales } \\
\hline Organización y planificación \\
\hline Comunicación oral y escrita \\
\hline Uso de las TIC \\
\hline Toma de decisiones \\
\hline Gestión de la información y el conocimiento \\
\hline Competencias personales \\
\hline Trabajo en equipo \\
\hline Interacción social \\
\hline Control emocional \\
\hline Competencias sistémicas \\
\hline Trabajo autónomo \\
\hline Actitud emprendedora \\
\hline Creatividad e innovación \\
\hline Adaptación \\
\hline Motivación \\
\hline Investigación \\
\hline
\end{tabular}

Fuente: Martínez Clares y González Morga (2019:9)

Por otra parte, queremos destacar la importancia de la innovación docente que implica que dos profesores expliquen a un mismo grupo la misma asignatura. Hasta ahora, lo más

${ }_{9}^{9}$ Dentro de las competencias instrumentales propuestas por Martínez Clares y González Morga (2019) no adquieren las relativas a las comunicación en lengua extranjera (es una asignatura impartida en español y con la bibliografía en español) y el diseño y gestión de proyectos profesionales y de vida y toma de decisiones. En competencias personales el compromiso ético profesional y responsabilidad social. 
frecuente era que un profesor impartiese la clase a su grupo, lo que le permitía una cierta autonomía respecto a otros profesores de la materia, descuidando la coordinación en algunos casos y haciendo que dos grupos distintos en una misma materia pudiesen llegar a tener programas casi diferentes. También era habitual el caso de dos profesores que impartían en un mismo grupo una misma materia, pero uno de ellos dedicado a las clases teóricas y otro a las prácticas, o que se repartían los temas por la especialidad de cada uno de ellos. En el caso de la asignatura Historia y Estructura Económica, los dos profesores imparten tanto la parte teórica, como la práctica de forma indistinta, y se ha diseñado el programa para potenciar la especialidad de cada uno de los profesores en cada uno de los temas, pero sin que haya una ruptura en el semestre. De esta forma, el temario analiza a la economía española desde principios del siglo XX hasta la actualidad, lo que obliga a que los temas sean impartidos por los dos profesores - Don Pedro Fernández, profesor de Historia Económica, y Doña Inmaculada Hurtado, profesora de Economía Española - a un mismo tiempo. Los profesores, desde esta perspectiva, adquieren un compromiso de responsabilidad del bienestar de los estudiantes y su aprendizaje. No se limitan, como señala Fernández Batanero (2004:8), a impartir una serie de contenidos inconexos y por tanto faltos de significatividad, constituyendo esta experiencia una alternativa a la fragmentación de la enseñanza.

Desde el punto de vista del alumno, se nos ha transmitido que hay una gran uniformidad en la materia y que las clases son más dinámicas, así como la mayor disponibilidad del profesorado para la resolución de sus dudas, dado que pueden optar por dos horarios a los que poder dirigir sus cuestiones. Desde la perspectiva de los profesores implicados, debemos destacar el aprendizaje mutuo que nos está reportando el poder explicar una materia como esta, desde una perspectiva distinta a la que hasta el momento estábamos acostumbrados.

Con este sistema de clases se demuestra la importancia de la transversalidad de las asignaturas. Uno de los objetivos planteados era que los alumnos fueran capaces de comprobar que las asignaturas que ellos estudian a lo largo de su vida universitaria no son asignaturas compartimentadas sin ninguna conexión entre ellas, sino que por el contrario existen grandes sinergias que deben aprovechar para que el resultado final de sus estudios universitarios pueda ser mucho mejor. Así, en la vida profesional pueden comprobar e interconectar conceptos que pudieran parecer que, en un principio, no tenían ninguna relación. Se trata, por tanto, de una materia con un carácter interdisciplinar - de hecho, es una asignatura que pertenece a dos Áreas de Conocimiento y a dos Unidades Docentes diferentes - y que cualifica mejor a los alumnos de cara a su futura inserción social y profesional.

El alumno sigue todo un proceso de evaluación continua que tiene como resultado un sistema que permite reflejar las competencias que ha adquirido con su aprendizaje en clase y su trabajo individual en casa. 


\section{Resultados y conclusiones: un nuevo modelo pedagógico ejemplo de transversalidad}

El modelo pedagógico que hemos planteado supone pues un sistema de clases donde la materia analizada tiene por objeto analizar la realidad actual, teniendo en cuenta todos los factores que han influido en el largo plazo. A lo largo del semestre, los profesores interactúan, permitiendo que los alumnos sean capaces de adquirir esa visión e incentivando la participación activa en todas las clases.

En definitiva se pretende que la transversalidad sea una evidencia, haciendo que la dinámica de la clase obligue a los dos profesores a "reeditarse" cada día, contando con lo que cada uno explica y con lo que se quiere obtener de cada actividad llevada a cabo. Por su parte, los estudiantes deben trabajar de forma individual para poder asimilar los conceptos y tener sus propias opiniones, pero también han de saber defender dichas ideas en grupos de trabajo para consensuar una postura común de cara a la práctica que entregan al finalizar el semestre.

Tabla 2. Resultado de las encuestas realizadas por los estudiantes a los dos profesores de la asignatura

\begin{tabular}{|c|c|c|c|c|c|c|}
\hline & \multicolumn{2}{|c|}{$1^{\infty x}$ año } & \multicolumn{2}{|c|}{$2^{\circ}$ año } & \multicolumn{2}{|c|}{$3^{a}$ año } \\
\hline & Estructura & Historia & Estructura & Historia & Es structura & Historia \\
\hline Grado de interés por la asignatura & 8,31 & 8,15 & 8,85 & 8,38 & & \\
\hline Tutorias académicas* & 6,6 & 9 & 9,14 & 9,25 & & \\
\hline Labor docente & 6,63 & 6,54 & 8,07 & 8,4 & 7,95 & 7,16 \\
\hline Rigor académico & 6,89 & 7,13 & 8,62 & 9,04 & 7,88 & 7,03 \\
\hline Cualidades docentes & 7,28 & 6,77 & 8,62 & 8,74 & 8,26 & 7,55 \\
\hline Metodologia y didactica & 7,08 & 6,64 & 8,29 & 8,59 & 7,68 & 7,21 \\
\hline \multirow[t]{3}{*}{ MEDIA DOCENTE } & 7.06 & 6.93 & 8.57 & 8.87 & 7.99 & 7.24 \\
\hline & \multicolumn{2}{|c|}{$4^{\circ}$ año } & \multicolumn{2}{|c|}{$5^{\circ}$ año } & & \\
\hline & Estructura & Historia & Estructura & Historia & & \\
\hline \multicolumn{7}{|l|}{$\begin{array}{l}\text { Grado de interés por la asignatura } \\
\text { Tutorias académicas }\end{array}$} \\
\hline Labor docente & 9,25 & 7,75 & 10 & 9,33 & & \\
\hline Rigor académico & 8,73 & 8,43 & 9,7 & 8,65 & & \\
\hline Cualidades docentes & 8,97 & 7,94 & 10 & 9,17 & & \\
\hline Metodologia y didactica & 8,38 & 6,38 & 9,75 & 9 & & \\
\hline MEDIA DOCENIE & 8.76 & 795 & 9.81 & 8.88 & & \\
\hline
\end{tabular}

- A partir del tercer año, la Universidad cambió las preguntas que se hacian a los estudiantes y se eliminaron éstas. Fuente: Blaboración propia

El resultado de todo ello es un elevado grado de satisfacción por parte de los alumnos con todo lo aprendido a lo largo del curso. Esto se desprende no sólo de las opiniones que nos han ido transmitiendo personalmente en las tutorías personales o grupales que hemos mantenido todos estos años con ellos, sino también por una muy aceptable valoración a ambos profesores durante todos estos años. Tal y como se puede observar en la tabla 2, donde aparecen las opiniones anónimas que realizan anualmente los alumnos sobre determinados 
aspectos relacionados con la docencia de cada profesor que les imparte clase, las valoraciones que han hecho a ambos profesores son muy parecidas durante los años analizados.

Además, a lo largo de estos años, la valoración de ambos profesores ha sido muy alta - por encima del 8,2 en promedio de los años analizados - lo que indica que el grado de satisfacción del alumnado con este modelo docente es elevado y no da señales para un abandono en los próximos cursos académicos. Asimismo, la similitud de notas a cada profesor también muestra los estudiantes no ven en el cambio entre uno y otro durante las distintas clases sea un inconveniente a la hora de estudiar la asignatura.

A modo de conclusión, podríamos decir que este nuevo proyecto pedagógico se basa en que dos profesores impartan clase al unísono, a un mismo grupo de alumnos la misma asignatura sin hacer una diferenciación temporal en el temario, por lo que las clases de uno y otro se van alternando según el tema va avanzando. Este carácter interdisciplinar ha supuesto un gran esfuerzo a la hora de elaborar todo el material docente - teórico y práctico - y en lo referido a la evaluación del alumno.

De gran ayuda es el manejo de las nuevas tecnologías, especialmente en la parte práctica de la materia, y la disponibilidad de la Blackboard como medio de transmisión y comunicación entre todos. Uno de los principales objetivos que se buscaba al desarrollar este nuevo modelo pedagógico era que la calidad de la docencia fuera muy superior a lo que podría haber sido con las clases tradicionales.

De esta forma, la coordinación entre los dos profesores se transmite como un valor esencial a los alumnos, para que ellos en su trabajo también sean capaces de colaborar y coordinarse con el resto de sus compañeros. Asimismo, los profesores hemos considerado que ellos deben ser conscientes de que el periodo de tiempo universitario debe servirles, no sólo para recibir una serie de conceptos básicos relativos a su carrera, sino como "laboratorio de ensayo" para su posterior vida laboral, en la que sean capaces de esforzarse y dar lo mejor de sí mismos; de cumplir con unos plazos establecidos para la entrega de trabajos e incluso la entrada en clase; la colaboración que con el resto de sus compañeros; el respeto de opiniones diferentes.

En definitiva, con una asignatura como Historia y Estructura Económica se ha conseguido que dos materias independientes puedan mostrarse como complementarias, gracias al trabajo continuo y la colaboración permanente entre los dos profesores, por un lado, y entre ellos y los alumnos, por otro, tal y como muestra la alta valoración de ambos docentes por parte de los alumnos en las encuestas anónimas de final de curso. Por tanto, utilizando la terminología propuesta por De la Herrán (2005:242), constituye un claro ejemplo de:

a) Coordinación interdisciplinar, a través de relaciones horizontales entre asignaturas simultáneas del mismo curso (en este caso convertidas en una única asignatura, llevando más allá el concepto de coordinación), y verticales con asignaturas de cursos anteriores como las Teorías Económicas I y II.

b) Profundización transdisciplinar desde relaciones tanto transversales a través de contenidos comunes abordables desde distintas asignaturas, como radicales, a partir de cada materia hacia contenidos perennes. 
De igual manera reúne las características de transversalidad universitaria propuestas por De la Herrán, Paredes Labra, Cuenca Escribano, Sánchez Grey, Cerrillo Martín, Del Hoyo y Veganzones Rueda (2003), ya que este proyecto docente:

a) Está protagonizado por profesores y estudiantes

b) Está abierto al diálogo, a la profundización y a una evaluación continua.

c) Es flexible, y se adapta a las circunstancias de los docentes (en función de la evolución del curso se deben ir adaptando a las circunstancias) así como de los estudiantes.

Por todo ello se considera muy conveniente, no sólo el mantenimiento de este tipo de experiencias, por lo que aportan tanto a nivel docente como educativo, sino también su extensión a otras asignaturas, dado el buen resultado obtenido.

\section{BIBLIOGRAFÍA:}

ANECA (2005). Libro Blanco, Título de Grado en Economía y en Empresa. Recuperado de http://www.aneca.es/Documentos-y-publicaciones/Libros-Blancos

Arroyo, MJ., Hurtado, I., Abeleira, C. y Requejo, P. (2011). "El alumno y su aprendizaje en el trabajo en grupo: una experiencia en el área de economía aplicada". En Canabal, C. y García, MD. (dtras.). La creación de espacios comunes de aprendizaje: Experiencias de innovación. Universidad de Alcalá, pp. 305-326.

Cátedra UNESCO (9 de Octubre de 1998). Declaración Mundial sobre la educación superior en el siglo XXI: visión y acción [página web]. Recuperado de http://www.unesco.org/education/educprog/wche/declaration_spa.htm

CEDEFOP (2014). Centro Europeo para el Desarrollo de la Formación Profesional: Desajuste de competencias: más de lo que parece a simple vista [nota informativa] Recuperado de http://www.cedefop.europa.eu/files/9087_es.pdf

De la Herrán, A. (2005). "Formación y transversalidad universitarias". Tendencias Pedagógicas, [S.1.], v. 10, p. 223-256.

De la Herrán, A. (coord.), Paredes Labra, J., Cuenca Escribano, A., Sánchez Grey, J., Cerrillo Martín, R., Del Hoyo Gómez, C. y Veganzones Rueda, J. (2003). Guías didácticas para la formación de maestros. Huelva: Hergué.

European Commission (2018). Promoting the relevance of higher education. Luxemburgo. Recuperado de https://publications.europa.eu/en/publication-detail/-/publication/59d3a99984b9-11e8-ac6a-01aa75ed71a1

Fernández Batanero, J.M. (2004). "La transversalidad curricular en el contexto universitario: un puente entre aprendizaje académico y el natural". Revista Fuentes, 5. Recuperado de https://revistascientificas.us.es/index.php/fuentes/article/view/2403 
«Haciendo de la coordinación una experiencia transversal: el estudio de la Estructura Económica en el siglo XXI desde la perspectiva del largo plazo»

Martínez Clares, P. y González Morga, N. (2019). "El dominio de competencia transversales en Educación Superior en diferentes contextos formativos". Educação e Pesquisa, v.45, pp. 1-23. Recuperado de http://www.scielo.br/pdf/ep/v45/1517-9702-ep-45-e188436.pdf

OIT (2017): Global employment trends for youth 2017 [documento online]. Recuperado de https://www.ilo.org/wcmsp5/groups/public/---dgreports/---dcomm/--publ/documents/publication/wcms 598669.pdf 